Article

\title{
Click Reactions as a Key Step for an Efficient and Selective Synthesis of D-Xylose-Based ILs
}

\author{
Nadège Ferlin ${ }^{1}$, Sylvain Gatard ${ }^{1}$, Albert Nguyen Van Nhien ${ }^{2}$, Matthieu Courty ${ }^{3}$ and \\ Sandrine Bouquillon ${ }^{1, *}$
}

1 Institut de Chimie Moléculaire de Reims, UMR CNRS 6229, Université de Reims

Champagne-Ardenne, Boîte 44, B.P. 1039, Reims F-51687, France;

E-Mails: nadege.ferlin@ipb.fr (N.F.); sylvain.gatard@univ-reims.fr (S.G.)

2 Laboratoire des Glucides FRE 3517, Université de Picardie Jules Verne, UFR des Sciences, 33 rue Saint Leu, Amiens Cedex 1 80039, France; E-Mail: albert.nguyen-van-nhien@u-picardie.fr

3 Laboratoire de Réactivité et de Chimie des Solides UMR CNRS 7314, Université de Picardie Jules Verne, UFR des Sciences, 33 rue Saint Leu, Amiens Cedex 180039 , France; E-Mail: matthieu.courty@u-picardie.fr

* Author to whom correspondence should be addressed; E-Mail: sandrine.bouquillon@univ-reims.fr; Tel.: +33-3-2691-8973; Fax: +33-3-2691-3166.

Received: 27 June 2013; in revised form: 7 August 2013 / Accepted: 10 September 2013 / Published: 17 September 2013

\begin{abstract}
D-Xylose-based ionic liquids have been prepared from D-xylose following a five steps reaction sequence, the key step being a click cycloaddition. These ionic liquids (ILs) have been characterized through classical analytical methods (IR, NMR, mass spectroscopy, elemental analysis) and their stability constants, Tg and Tdec, were also determined. Considering their properties and their hydrophilicity, these compounds could be alternative solvents for chemical applications under mild conditions.
\end{abstract}

Keywords: ionic liquids; D-xylose; click chemistry

\section{Introduction}

In the last two decades, ionic liquids (ILs) have attracted considerable attention due to their unique properties (non-flammability, good electrolytic properties, unique solubility, negligible vapor pressure, good thermal stability, etc.) [1-3]. Due to the increasing growth of their applications as 
alternative to volatile solvents in catalytic applications [1,4-6], biocatalysis [7,8], synthetic chemistry [9], electrochemistry [10-14], analytical applications [15-19], or for separations and extractions [20-26], the development of new IL structures is always being sought. Thanks to the click chemistry reaction, a large variety of 1,2,3-triazole structures can be obtained [27-29], but surprisingly, few ionic liquids derived from triazole have been reported (Figure 1) [30-34].

Figure 1. Triazolium based ionic liquids [13].
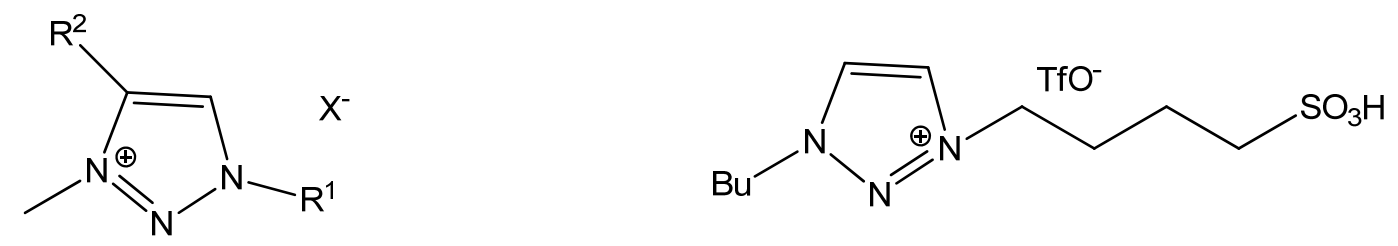

$\mathrm{R}^{1}=\mathrm{Ph}, \mathrm{Bn}, \mathrm{Me}$, allyl; $\mathrm{R}^{2}=\mathrm{H}, \mathrm{Bu}, \mathrm{Pn}, \mathrm{Hex}, t$-Bu, Ph

$\mathrm{X}=\mathrm{OTf}, \mathrm{Tos}, \mathrm{I}, \mathrm{BF}_{4}, \mathrm{PF}_{6}, \mathrm{NTf}_{2}$

Carbohydrates are among the most abundant and low-cost natural sources of chiral materials and represent building blocks of choice for the formation of various compounds with a broad spectrum of applications [35]. The use of ILs as solvents for the transformation of carbohydrates was first reviewed by Linhardt in 2005 [36]. Next, ILs have been shown to exhibit excellent solubilizing properties, facilitating a wide range of chemical transformations, including acetylation, ortho-esterification, benzylidenation and glycosylation reactions of carbohydrates [36-41]. Recently, Afonso and Tran discussed respectively the application of ILs in carbohydrate dissolution [42] and the recent developments of ionic liquids in oligosaccharide synthesis [43]. Therefore, sugar-based chiral ionic liquids (CILs) could be used as solvent or catalyst in asymmetric synthesis [44-48] or as chiral phases in gas chromatography [49].

Only a few examples of carbohydrates-based ILs were reported in the literature [50-57] (Figure 2). First, in 2003 Dickenson et al. published the preparation of ILs derived from fructose as a promising solvent for implementing fully "green chemistry" methods [50]. Glucose was also used as starting material for the elaboration of either a new class of chiral solvents from low-cost natural sources [51] or multiphase particles for cosmetic applications [52]. Next, isomanide or isosorbide-based ILs were prepared as solvents for chiral discrimination or asymmetric organic reactions [53-57].

For our part, we recently reported the preparation (and the use as solvent for catalysis) of biomassderived ionic liquids from natural organic acids, among them osidic acids [58] (Figure 3). In this context, as we have been studying for many years the valuation of pentoses issued from hemicelluloses as surfactants [59-64] or glycodendrimers [65-67], we wish to report here a new way of valuation of these sugars as new ILs in which 1,2,3-triazolium salts [33,34,68-76] serve as the IL part and xyloside units are covalently tethered at the " 4 " position of the triazolium ring. To the best of our knowledge, no ionic liquid derived from D-xylose was previously described in the literature. 
Figure 2. Carbohydrates-based ILs.

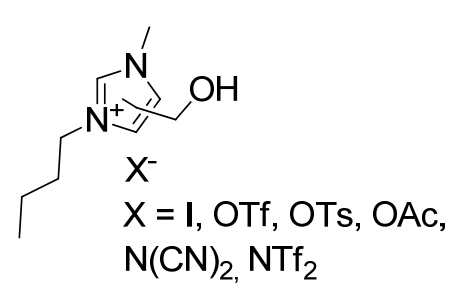

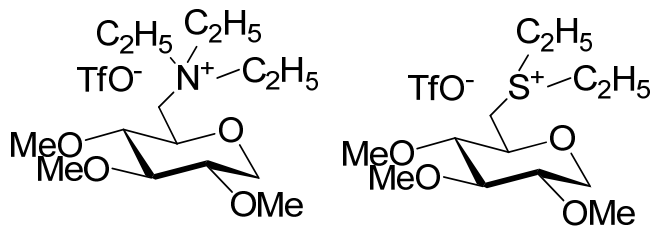

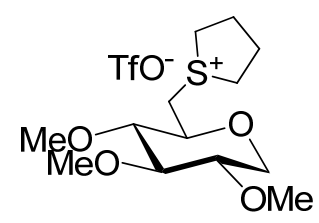

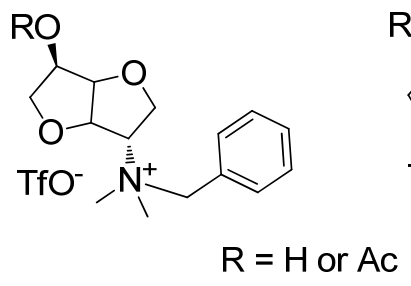
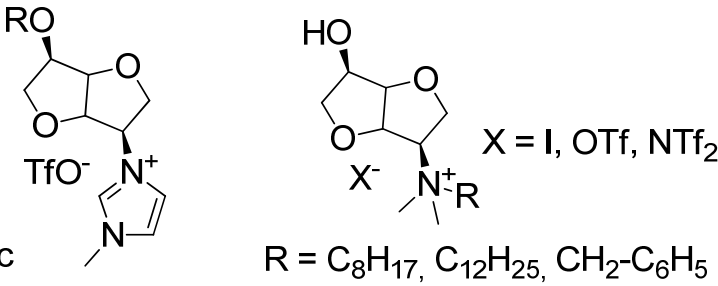

Figure 3. Biomass derived ILs.

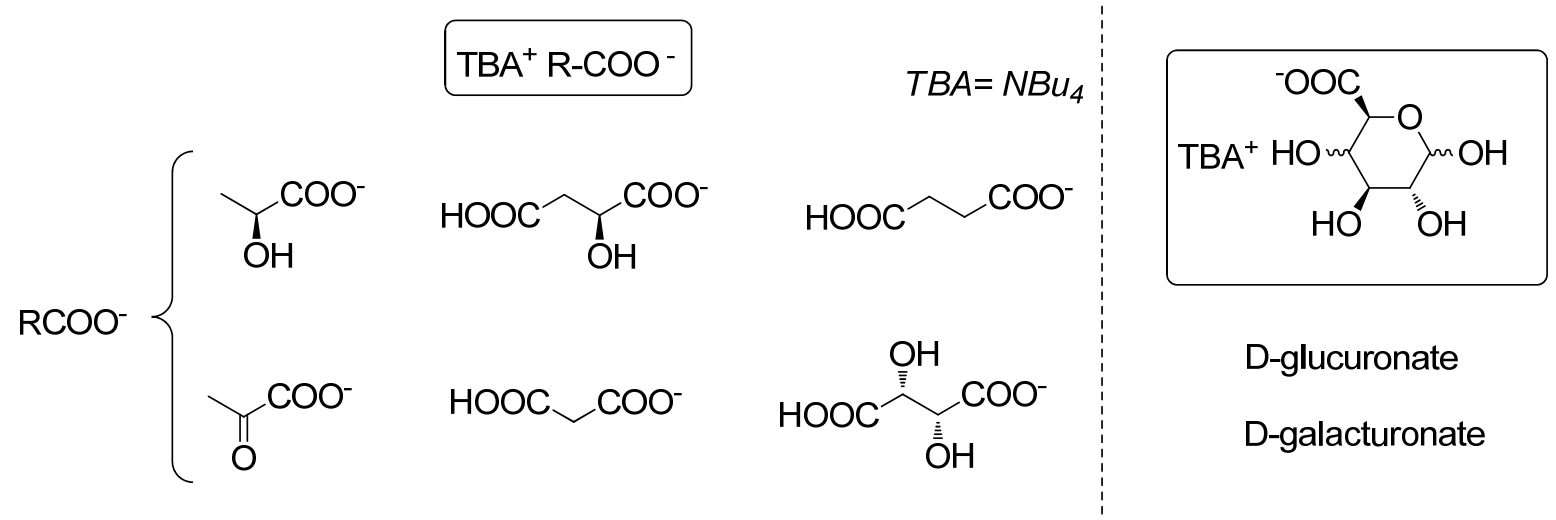

\section{Results and Discussion}

For the glycosylation step, treatment of peracetylated D-xylose with propargyl alcohol in the presence of $\mathrm{BF}_{3} \cdot \mathrm{Et}_{2} \mathrm{O}$ was used to access the $\beta$-propargyl xyloside 1 [77]. This method was preferred because previous trials on D-xylose using the Fisher method [78] with para-toluenesulfonyl acid as catalyst led to a mixture of anomers which are could not be separated, even after acetylation.

$\mathrm{Cu}^{\mathrm{I}}$-"catalyzed" Huisgen 1,3-dipolar cycloaddition reaction of the modified alkynyl sugar with phenyl or hexyl azide, was carried out in the presence of an excess of $\mathrm{Cu}^{\mathrm{I}}$ in a homogeneous THF/water mixture (Scheme 1). Several reactions were performed with catalytic and stoichiometric amounts of copper, but led to very poor yields, a part of the copper salt probably being involved in the complexation of the acetate groups. The propargyl xyloside/azide ratio was also optimized after several trials to afford good yields for the cycloaddition adducts.

The excess of $\mathrm{Cu}$ salt was removed as $\left[\mathrm{Cu}\left(\mathrm{NH}_{3}\right)_{2}\left(\mathrm{H}_{2} \mathrm{O}\right)_{2}\right]\left[\mathrm{SO}_{4}\right]$ by washing with an ammonia solution. Purification by precipitation with $\mathrm{CH}_{2} \mathrm{Cl}_{2}$ /petroleum ether in order to remove the excess of sugar provided compounds $\mathbf{2}$ and $\mathbf{3}$ in good yields. The presence of signals at $7.42 \mathrm{ppm}$ and $7.49 \mathrm{ppm}$ for 2 and 3, respectively, in their ${ }^{1} \mathrm{H}-\mathrm{NMR}$ spectrum, unambiguously proved the formation of the triazole ring. The composition of compounds $\mathbf{2}$ and $\mathbf{3}$ was further confirmed by ${ }^{13} \mathrm{C}-\mathrm{NMR}$ and 
elemental analysis. The acetylated benzyl and hexyl compounds $\mathbf{2}$ and $\mathbf{3}$ were then deprotected in the presence of sodium methanolate to give the corresponding derivatives $\mathbf{4}$ and $\mathbf{5}$ with free hydroxyl groups (Scheme 1). No signals were found for methyl groups or carbonyl carbons in the ${ }^{1} \mathrm{H}-$ and ${ }^{13} \mathrm{C}-\mathrm{NMR}$ spectra, respectively. This set of derivatives was purified by precipitation.

Scheme 1. Synthesis of ILs 6 and 7.

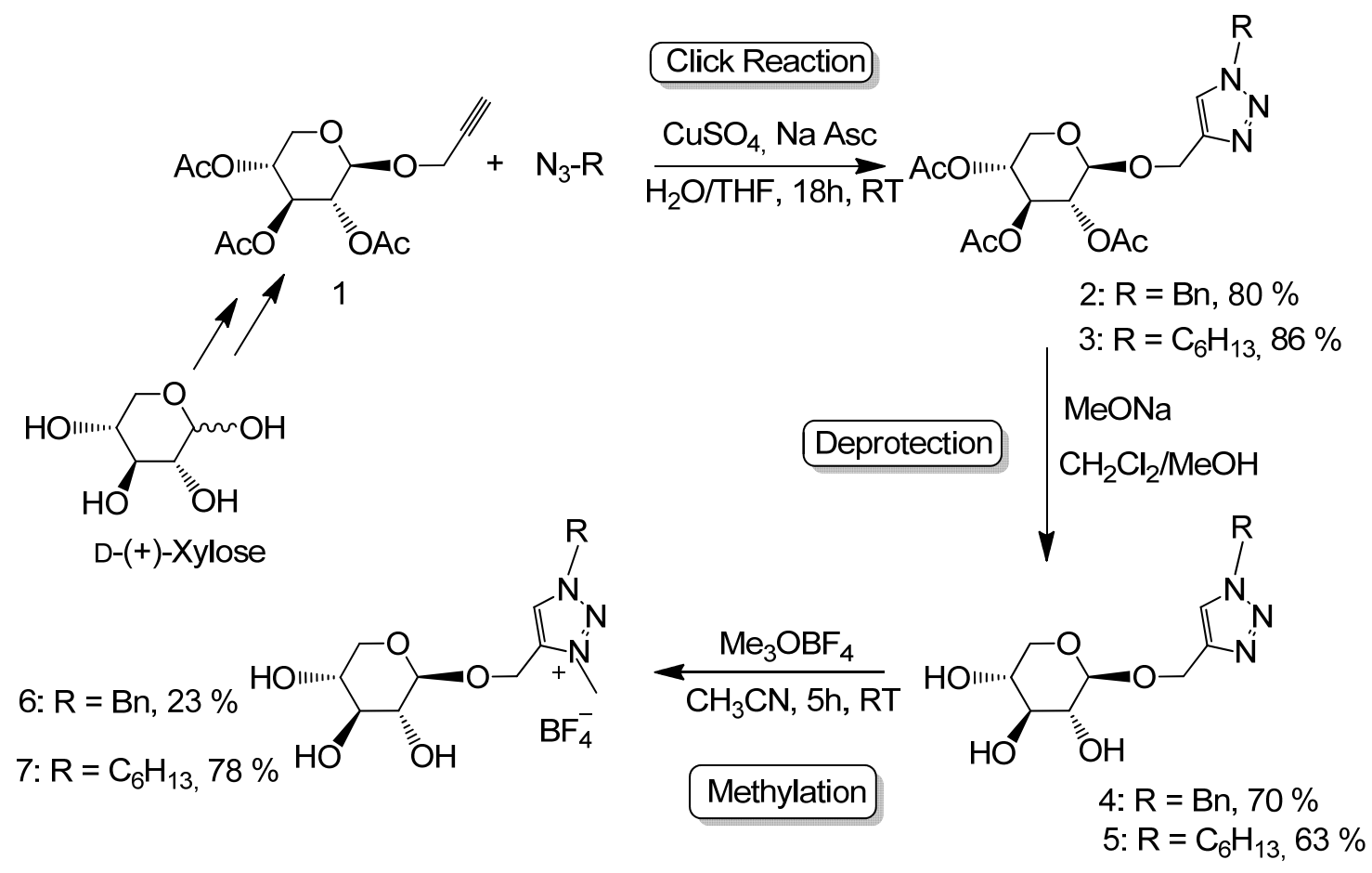

In line with previous observations, trimethyloxonium tetrafluoroborate (Meerwein's salt) proved to be a very powerful methylating agent (29 equivalents used as described [79]), affording benzyl and hexyl triazolium salts 6 and 7 in good isolated yields in $5 \mathrm{~h}$ at room temperature in dry MeCN (Scheme 1). Alternative reaction conditions applied to the hexyl derivative, using methyl iodide (20 equivalents) in dry $\mathrm{MeCN}$ under reflux gave improved yields $(95 \%)$ but required longer reaction times $(85 \mathrm{~h})$. The new ILs 6 and 7 were highly soluble in water and in methanol and insoluble in diethyl ether, therefore their purification was done by precipitation of the crude products from $\mathrm{MeOH} / \mathrm{Et}_{2} \mathrm{O}$. The presence of signals around $4.32 \mathrm{ppm}$ in their ${ }^{1} \mathrm{H}-\mathrm{NMR}$ spectrum and at $38.7 \mathrm{ppm}$ in their ${ }^{13} \mathrm{C}$-NMR spectrum for the benzyl and hexyl derivatives, respectively, showed the quaternisation of the triazole ring.

In addition of the IR, NMR, elemental analyses and mass spectroscopy, ILs 6 and 7 were characterized by DSC (Table 1) and TGA (Figure 4). Both compounds are stable until $120{ }^{\circ} \mathrm{C}$ and $150{ }^{\circ} \mathrm{C}$, respectively, and showed a slight positive glass transition temperature $(\mathrm{Tg})$. As previously described for tetrabutylammonium galacturonate and glucuronate [58], positive $\mathrm{Tg}$ and low decomposition temperature are observed what seems to be in relation with the presence of sugar moities. Considering these temperatures, $\mathbf{6}$ and $\mathbf{7}$ could be used only under mild conditions as solvents or chiral agents for chemical transformations or catalysis. 
Table 1. Glass transition and decomposition temperatures of ILs 6 and 7.

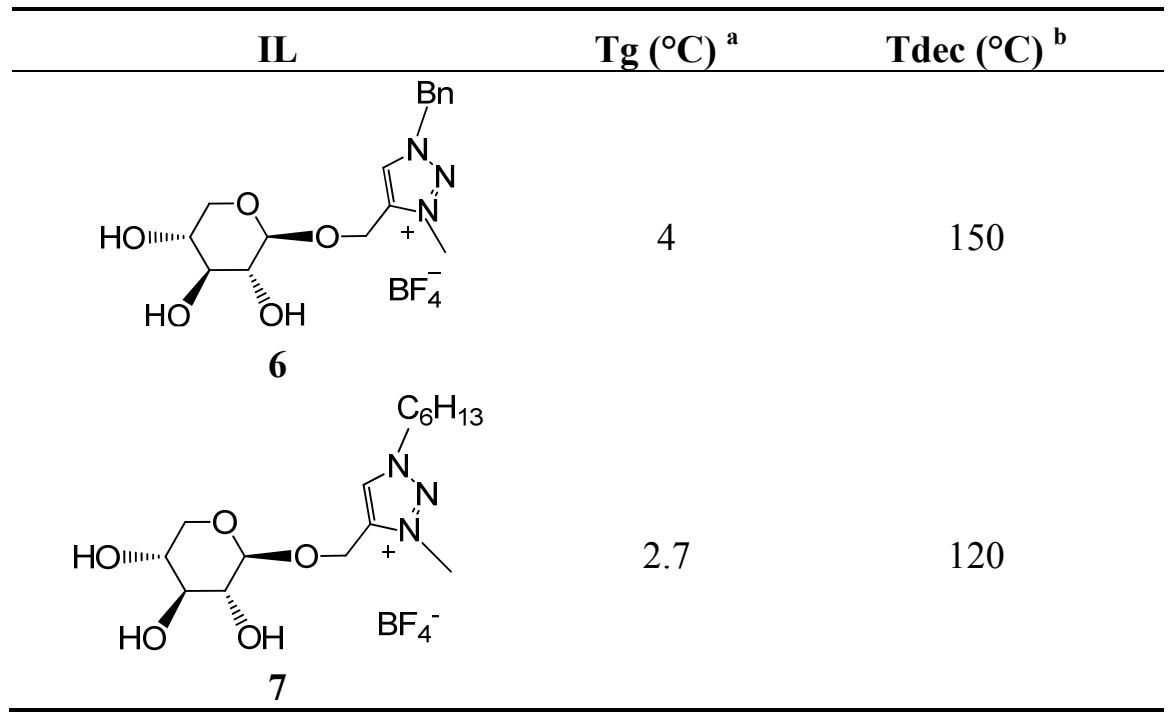

${ }^{\mathrm{a}} \mathrm{Tg}=$ Onset temperature measured at $10 \mathrm{~K} / \mathrm{min}$ under argon; ${ }^{\mathrm{b}} \mathrm{Tdec}=$ Onset temperature measured at $10 \mathrm{~K} / \mathrm{min}$ under argon.

Figure 4. Thermogravimetric analysis of compounds 6 and 7.

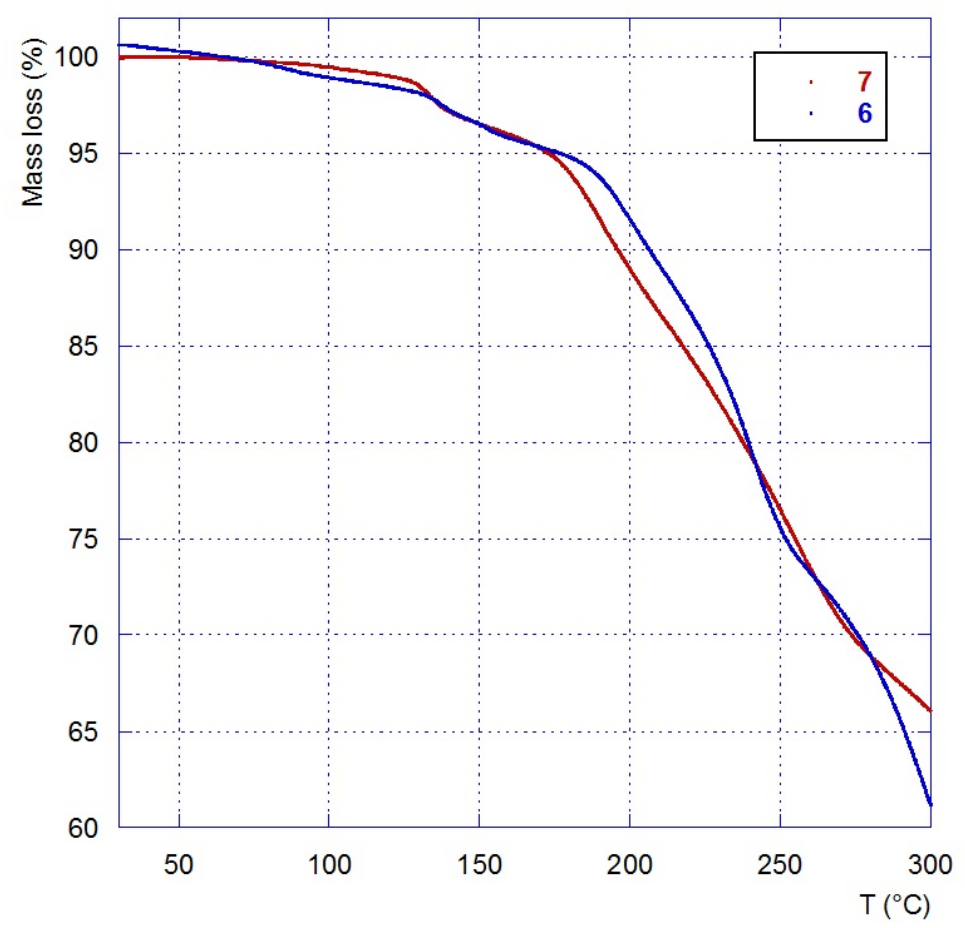

The thermal stability of $\mathbf{6}$ and 7 was determined by thermogravimetric analysis (TGA) under argon (Figure 4). The TG curve shows an initial weight loss of $1.33 \%$ and $0.76 \%$ of water respectively for 6 and 7 between room temperature and $110{ }^{\circ} \mathrm{C}$ followed by a second loss of water $(3.20 \%$ and $3.01 \%)$. Such a noticeable mass loss corresponds to the hydroxyl groups. The thermal degradation (Tdec) occurring during the second step gives a loss of $\mathrm{F}(\mathrm{m} / \mathrm{z}=19)$ fragments by mass spectrometry analysis originating from $\mathrm{BF}_{4}{ }^{-}$decomposition. 


\section{Experimental}

\subsection{General Procedures}

All reagents were commercially available and used as received. $\mathrm{CH}_{2} \mathrm{Cl}_{2}$ was dried over $\mathrm{CaH}_{2}$ and distilled under argon before use. $\mathrm{CH}_{3} \mathrm{CN}$ was dried using a Pure Solv solvent drying system over aluminum oxide under an argon atmosphere before use. ${ }^{1} \mathrm{H}-\mathrm{NMR}(250.1 \mathrm{MHz}),{ }^{13} \mathrm{C}-\mathrm{NMR}(62.9 \mathrm{MHz})$ and ${ }^{19} \mathrm{~F}-\mathrm{NMR}(235.4 \mathrm{MHz})$ spectra were recorded on an $\mathrm{AC} 250$ Bruker instrument in $\mathrm{CDCl}_{3}$ or $\mathrm{MeOD}$ with TMS as reference for ${ }^{1} \mathrm{H}$ spectra and $\mathrm{CDCl}_{3}(\delta 77.0)$ or $\mathrm{MeOD}(\delta 49.9)$ for ${ }^{13} \mathrm{C}$ spectra. IR spectra were recorded on a Nicolet AVATAR 320 FT-IR. C and H analyses were performed on a Perkin Elmer $2400 \mathrm{CHN}$ equipment. Chromatographies were carried out on SDS Silica $60(40-63 \mu \mathrm{m})$ or Silica $60 \mathrm{~F}_{254}$ (TLC plates). All experiments (MS and HRMS) were obtained on a hybrid tandem quadrupole/time-of-flight (Q-TOF) instrument, equipped with a pneumatically assisted electrospray (Z-spray) ion source (Micromass, Manchester, UK) operated in positive and negative mode. The electrospray potential was set to $3 \mathrm{kV}$ in positive ion mode (flow of injection $5 \mu \mathrm{L} / \mathrm{min}$.) and the extraction cone voltage was usually varied between 30 and $90 \mathrm{~V}$. Optical rotations were measured on a Perkin Elmer 241 polarimeter. Thermogravimetric analyses coupled with a mass spectrometer were performed between $30{ }^{\circ} \mathrm{C}$ and $300{ }^{\circ} \mathrm{C}$ under a constant flow of dry argon $\left(50 \mathrm{~mL} \cdot \mathrm{min}^{-1}\right)$ using a Simultaneous Thermal Analyzer STA 449C Jupiter from Netzsch, and a heating rate of $10 \mathrm{~K} / \mathrm{min}$. The isothermal drift and sensitivity values are $0.6 \mu \mathrm{g} / \mathrm{h}$ and $0.1 \mu \mathrm{g}$, respectively. Alumina crucibles were loaded with 10-20 mg of sample. The DSC experiments were carried out on a Netzsch DSC 204F1 heat flux differential calorimeter at a heating rate of $10 \mathrm{~K} /$ min under a constant flow of dry argon $\left(200 \mathrm{~mL} \cdot \mathrm{min}^{-1}\right)$. Aluminum crucibles were loaded with $10-15 \mathrm{mg}$ of sample.

\subsection{Synthetic Procedures}

3.2.1. Preparation of 1-((1-Benzyl-1,2,3-triazol-4-yl)methoxy)2,3,4-tri- $O$-acetyl- $\beta$-D-xylopyranoside (2)

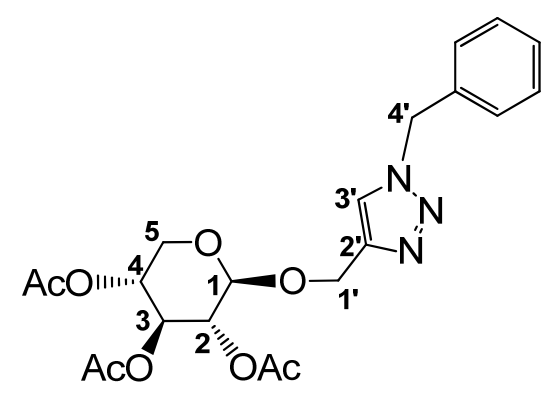

To a solution of $\beta$-propargyl xyloside $1(2.08 \mathrm{~g}, 6.3 \mathrm{mmol})$ in a THF/water 1:1 (v:v) (20 $\mathrm{mL})$ mixture were added benzyl azide $(560 \mathrm{mg}, 4.2 \mathrm{mmol}), \mathrm{CuSO}_{4} \cdot 5 \mathrm{H}_{2} \mathrm{O}(4.2 \mathrm{~g}, 16.9 \mathrm{mmol})$, and sodium ascorbate $(3.3 \mathrm{~g}, 16.9 \mathrm{mmol})$. The mixture was stirred at room temperature under an argon atmosphere for $18 \mathrm{~h}$. The mixture was concentrated and $\mathrm{CH}_{2} \mathrm{Cl}_{2}$ was added. The organic layer was washed with aqueous ammonium hydroxide $(0.8 \mathrm{M})$ until a colorless aqueous layer was obtained, then with water to neutrality. The organic phase was concentrated to dryness in vacuo. The crude product was dissolved in a minimum of $\mathrm{CH}_{2} \mathrm{Cl}_{2}$ and precipitated with an excess of petroleum ether. Compound 2 was obtained as a white solid in $80 \%$ yield $(2.25 \mathrm{~g})$. IR (KBr) $v \mathrm{~cm}^{-1}: 2959,2876,1755,1652,1487,1456$, 
1371, 1224, 1172, 1123, 1046. ${ }^{1} \mathrm{H}-\mathrm{NMR}\left(\mathrm{CDCl}_{3}\right) 1.82,1.91,1.96\left(3 \times \mathrm{s}, 9 \mathrm{H}, \mathrm{CH}_{3 \mathrm{Ac}}\right), 3.37(\mathrm{dd}$, $\left.J=9 \mathrm{~Hz}, J=11.7 \mathrm{~Hz}, 1 \mathrm{H}, H_{5}\right), 4.11\left(\mathrm{dd}, J=5 \mathrm{~Hz}, J=11.7 \mathrm{~Hz}, 1 \mathrm{H}, H_{5}\right), 4.61\left(\mathrm{~d}, J=5 \mathrm{~Hz}, 1 \mathrm{H}, H_{1 \beta}\right)$, 4.73-4.97 (overlap, $\left.2 \mathrm{H}+1 \mathrm{H}+1 \mathrm{H}, H_{1},+H_{2}+H_{4}\right), 5.14\left(\mathrm{t}, J=8.5 \mathrm{~Hz}, 1 \mathrm{H}, H_{3}\right), 5.52\left(\mathrm{~s}, 2 \mathrm{H}, H_{4}\right.$ ) ), 7.26-7.42 (overlapped, 5H, $\left.H_{\text {arom }}\right), 7.42\left(\mathrm{~s}, 1 \mathrm{H}, H_{3}\right)$ ). ${ }^{13} \mathrm{C}-\mathrm{NMR}\left(\mathrm{CDCl}_{3}\right) 20.4,20.5,20.6\left(\mathrm{CH}_{3 \mathrm{Ac}}\right), 54.0$ $\left(C_{1}{ }^{\prime}\right), 61.9,62.3\left(C_{5}, C_{4}\right), 68.7,70.5,71.2\left(C_{2}, C_{3}, C_{4}\right), 99.6\left(C_{1 \beta}\right), 122.6\left(C_{3}\right), 128.1,128.7,129.0$ $\left(\mathrm{CH}_{\text {arom }}\right), 134.5\left(C \mathrm{q}_{\text {arom }}\right), 144.5\left(C_{2},\right), 169.3,169.7,169.8\left(C=\mathrm{O}_{\mathrm{Ac}}\right)$. Anal. Found $(\mathrm{Calcd})$ for

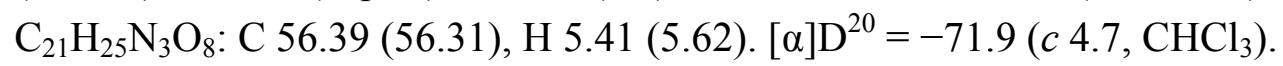

3.2.2. Preparation of 1-((1-Hexyl-1,2,3-triazol-4-yl)methoxy)2,3,4-tri- $O$-acetyl- $\beta$-D-xylopyranoside (3)

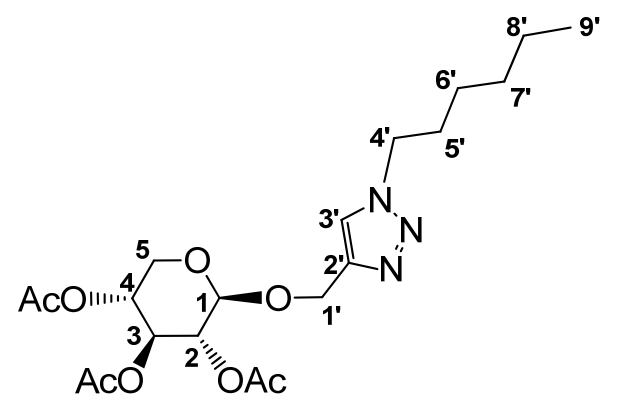

Same procedure as described for compound $\mathbf{2}$ was followed with a solution of $\beta$-propargyl xyloside $1(3 \mathrm{~g}, 9.5 \mathrm{mmol})$ in a THF/water $1: 1(\mathrm{v}: \mathrm{v})(20 \mathrm{~mL})$ mixture, hexyl azide (809 $\mathrm{mg}, 6.4 \mathrm{mmol})$, $\mathrm{CuSO}_{4} \cdot 5 \mathrm{H}_{2} \mathrm{O}(6.5 \mathrm{~g}, 26.0 \mathrm{mmol})$, and sodium ascorbate $(5.1 \mathrm{~g}, 26.0 \mathrm{mmol})$. Compound 3 was obtained as a white solid in $86 \%$ yield $(2.34 \mathrm{~g})$. IR $(\mathrm{KBr}) \mathrm{v} \mathrm{cm}^{-1}: 2957,2870,1758,1637,1464,1435,1228$, 1122, 1046. ${ }^{1} \mathrm{H}-\mathrm{NMR}\left(\mathrm{CDCl}_{3}\right) 0.85\left(\mathrm{~m}, 3 \mathrm{H}, H_{9}\right), 1.30$ (overlapped, $6 \mathrm{H}, H_{6},+H_{7},+H_{8}$ ) $), 1.90(\mathrm{~m}, 2 \mathrm{H}$, $H_{5}$ ) $, 2.01,2.03,2.05\left(3 \times \mathrm{s}, 9 \mathrm{H}, \mathrm{CH}_{3 \mathrm{Ac}}\right), 3.40\left(\mathrm{dd}, J=9 \mathrm{~Hz}, J=11.2 \mathrm{~Hz}, 1 \mathrm{H}, H_{5}\right), 4.15(\mathrm{dd}, J=5 \mathrm{~Hz}$, $\left.J=11.7 \mathrm{~Hz}, 1 \mathrm{H}, H_{5}\right), 4.35\left(\mathrm{t}, J=7.5 \mathrm{~Hz}, H_{4}{ }^{\prime}\right), 4.64\left(\mathrm{~d}, J=6.5 \mathrm{~Hz}, 1 \mathrm{H}, H_{1 \beta}\right), 4.89-4.97$ (overlapped, $\left.2 \mathrm{H}+1 \mathrm{H}+1 \mathrm{H}, H_{1},+H_{2}+H_{4}\right), 5.17\left(\mathrm{t}, J=10 \mathrm{~Hz}, 1 \mathrm{H}, H_{3}\right), 7.49\left(\mathrm{~s}, 1 \mathrm{H}, H_{3}\right)$. NMR ${ }^{13} \mathrm{C}(62.9 \mathrm{MHz}$, $\left.\mathrm{CDCl}_{3}\right) 13.8\left(C_{9}\right), 20.5\left(\mathrm{CH}_{3 \mathrm{Ac}}\right), 22.9,26.0,30.1,30.9\left(C_{5}, C_{6}, C_{7}, C_{8}\right), 50.2\left(C_{1}\right), 61.9,62.4\left(C_{5}\right.$, $\left.C_{4}\right)$, 68.7, 70.6, $71.2\left(C_{2}, C_{3}, C_{4}\right), 99.6\left(C_{1 \beta}\right), 122.3\left(C_{3}\right), 144.0\left(C_{2},\right), 169.3,169.7,169.8\left(C=\mathrm{O}_{\mathrm{Ac}}\right)$. Anal. Found (Calcd) for $\mathrm{C}_{20} \mathrm{H}_{31} \mathrm{~N}_{3} \mathrm{O}_{8}$ : C 54.34 (54.41), $\mathrm{H} 7.01$ (7.08). $[\alpha]_{\mathrm{D}}{ }^{20}=-67.0\left(c 4.2, \mathrm{CHCl}_{3}\right)$.

3.2.3. Preparation of 1-((1-Benzyl-1,2,3-triazol-4-yl)methoxy) $\beta$-D-xylopyranoside (4)

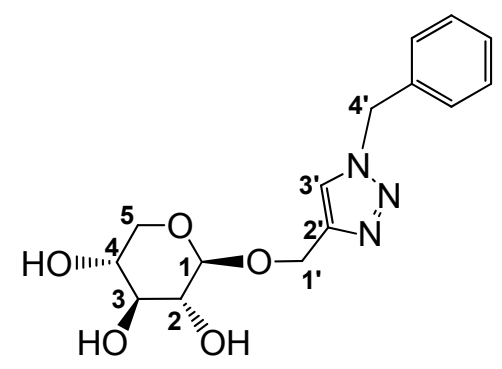

The acetylated compound 2 (170 mg, $0.38 \mathrm{mmol})$ was dissolved in $\mathrm{CH}_{2} \mathrm{Cl}_{2} / \mathrm{MeOH} \mathrm{1:1} \mathrm{(v:v)} \mathrm{(5} \mathrm{mL)}$ under $\mathrm{Ar}$ and $\mathrm{NaOMe}(61.8 \mathrm{mg}, 1.14 \mathrm{mmol})$ was then added. After stirring for $24 \mathrm{~h}$ at room temperature, the mixture was neutralized with Amberlite IR120 and filtered. The organic phase was concentrated to dryness in vacuo. The crude product was dissolved in a minimum of $\mathrm{MeOH}$ and precipitated with an excess of diethylether. Compound 4 was obtained as a white solid in $70 \%$ yield 
$(\mathrm{m}=85 \mathrm{mg}) .{ }^{1} \mathrm{H}-\mathrm{NMR}\left(\mathrm{CD}_{3} \mathrm{OD}\right) .3 .13-3.28$ (overlapped, $\left.1 \mathrm{H}+1 \mathrm{H}+1 \mathrm{H}, H_{2}+H_{3}+H_{5}\right), 3.44(\mathrm{~m}, 1 \mathrm{H}$, $\left.H_{4}\right), 3.82\left(\mathrm{dd}, J=5 \mathrm{~Hz}, J=11.2 \mathrm{~Hz}, H_{5}\right), 4.26\left(\mathrm{~d}, J=7.5 \mathrm{~Hz}, 1 \mathrm{H}, H_{1 \beta}\right), 4.67\left(\mathrm{~d}, J=12.5 \mathrm{~Hz}, H_{1}\right), 4.87$ (overlap, $\left.3 \mathrm{H}+1 \mathrm{H}, \mathrm{OH}+H_{1}{ }^{\prime}\right), 5.56\left(\mathrm{~s}, 2 \mathrm{H}, H_{4}\right), 7.30\left(\mathrm{~m}, 5 \mathrm{H}, H_{\text {arom }}\right), 7.94\left(\mathrm{~s}, 1 \mathrm{H}, H_{3^{\prime}}\right) .{ }^{13} \mathrm{C}-\mathrm{NMR}$ $\left(\mathrm{CD}_{3} \mathrm{OD}\right) 54.7\left(C_{1}\right), 62.8\left(C_{4}\right), 66.7\left(C_{5}\right), 70.9,74.5,77.3\left(C_{2}, C_{3}, C_{4}\right), 104.0\left(C_{1 \beta}\right), 125.1\left(C_{3}{ }^{\prime}\right), 128.9$, 129.4, $129.8\left(\mathrm{CH}_{\text {arom }}\right), 136.5\left(\mathrm{Cq}_{\text {arom }}\right), 145.7\left(C_{2}\right)$. Anal. Found (Calcd) for $\mathrm{C}_{15} \mathrm{H}_{19} \mathrm{~N}_{3} \mathrm{O}_{5}$ : $\mathrm{C} 55.97$ (56.03), H 5.94 (5.92). $[\alpha]_{\mathrm{D}}{ }^{20}=-36.7\left(c 6.0, \mathrm{H}_{2} \mathrm{O}\right)$.

3.2.4. Preparation of 1-((1-Hexyl-1,2,3-triazol-4-yl)methoxy) $\beta$-D-xylopyranoside (5)

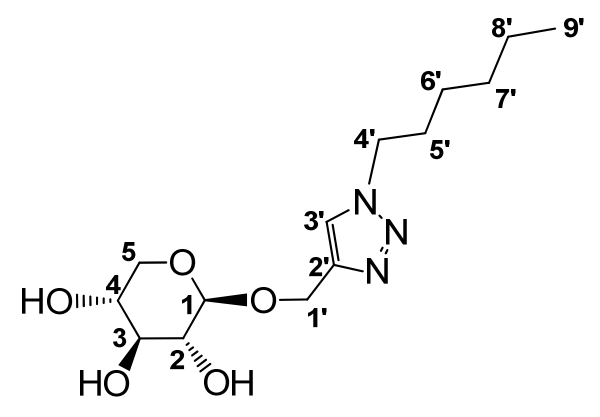

The same procedure as described for compound 4 was followed with compound 3 (2.34 g, $5.5 \mathrm{mmol}$ ) dissolved in $\mathrm{CH}_{2} \mathrm{Cl}_{2} / \mathrm{MeOH} \mathrm{1:1} \mathrm{(v:v)} \mathrm{(40} \mathrm{mL)} \mathrm{and} \mathrm{NaOMe}(887 \mathrm{mg}, 16.4 \mathrm{mmol})$. The compound 5 was obtained as a white solid in $63 \%$ yield $(\mathrm{m}=1.09 \mathrm{~g}) .{ }^{1} \mathrm{H}-\mathrm{NMR}\left(\mathrm{CD}_{3} \mathrm{OD}\right) 0.88(\mathrm{~m}, 3 \mathrm{H}$, $H_{9}$ ), 1.32 (overlapped, $6 \mathrm{H}, H_{6},+H_{7},+H_{8}$ ) $, 1.88\left(\mathrm{~m}, 2 \mathrm{H}, H_{5}{ }^{\prime}\right), 3.18-3.31$ (overlapped, $1 \mathrm{H}+1 \mathrm{H}+1 \mathrm{H}$, $\left.H_{2}+H_{3}+H_{5}\right), 3.48\left(\mathrm{~m}, 1 \mathrm{H}, H_{4}\right), 3.87\left(\mathrm{dd}, J=5 \mathrm{~Hz}, J=11.2 \mathrm{~Hz}, 1 \mathrm{H}, H_{5}\right), 4.30(\mathrm{~d}, J=7.5 \mathrm{~Hz}, 1 \mathrm{H}$, $\left.H_{1 \beta}\right), 4.38\left(\mathrm{t}, J=7.5 \mathrm{~Hz}, 1 \mathrm{H}, H_{4}\right), 4.70\left(\mathrm{~d}, J=12.5 \mathrm{~Hz}, 1 \mathrm{H}, H_{1}\right.$ ), 4.87 (overlapped, $3 \mathrm{H}+1 \mathrm{H}$, $\left.\mathrm{O} H+H_{1}{ }^{\prime}\right), 7.97\left(\mathrm{~s}, 1 \mathrm{H}, H_{3}{ }^{\prime}\right) .{ }^{13} \mathrm{C}-\mathrm{NMR}\left(\mathrm{CD}_{3} \mathrm{OD}\right) 13.9\left(C_{9^{\prime}}\right), 22.0,25.5,29.7,30.6\left(C_{5}, C_{6}, C_{7}, C_{8^{\prime}}\right)$, $49.3\left(C_{1}\right), 61.5\left(C_{4^{\prime}}\right), 65.8\left(C_{5}\right), 69.6,73.2,76.6\left(C_{2}, C_{3}, C_{4}\right), 102.8\left(C_{1 \beta}\right), 124.0\left(C_{3^{\prime}}\right), 143.6\left(C_{2}\right)$. Anal. Found (Calcd) for $\mathrm{C}_{14} \mathrm{H}_{25} \mathrm{~N}_{3} \mathrm{O}_{5}$ : C $53.28(53.32), \mathrm{H} 7.88(7.99)$. $[\alpha]_{\mathrm{D}}{ }^{20}=-40.0\left(c 2.4, \mathrm{H}_{2} \mathrm{O}\right)$.

3.2.5. Preparation of 1-((1-Benzyl-3-methyl-1,2,3-triazol-4-yl)methoxy) $\beta$-D-xylopyranoside tetrafluoroborate (6)

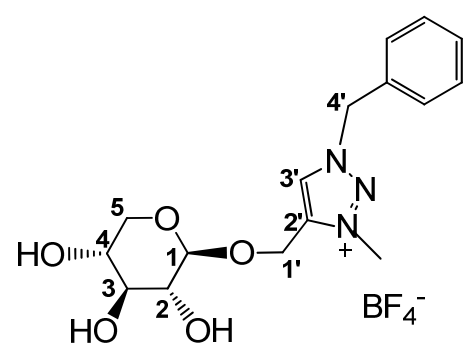

The corresponding triazole 4 (936 mg, $2.9 \mathrm{mmol})$ and $\mathrm{Me}_{3} \mathrm{OBF}_{4}(517 \mathrm{mg}, 3.5 \mathrm{mmol})$ were stirred in dry acetonitrile $(40 \mathrm{~mL})$ for $5 \mathrm{~h}$ at room temperature. The reaction was quenched with $\mathrm{MeOH}(10 \mathrm{~mL})$, and the solvent was removed under reduced pressure to give the crude product, which was in a minimum of $\mathrm{MeOH}$ and precipitated with excess of diethyl ether. Compound $\mathbf{6}$ was obtained as a white wax in $23 \%$ yield $(\mathrm{m}=291 \mathrm{mg})$. IR: $v \mathrm{~cm}^{-1}: 3363,2891,1737,1635,1589,1456,1348,1286$, 1244, 1155, 1035. ${ }^{1} \mathrm{H}-\mathrm{NMR}\left(\mathrm{CD}_{3} \mathrm{OD}\right)$ 3.13-3.29 (overlapped, $\left.1 \mathrm{H}+1 \mathrm{H}+1 \mathrm{H}, H_{2}+H_{3}+H_{5}\right), 3.36(\mathrm{~m}$, $\left.1 \mathrm{H}, H_{4}\right), 3.85\left(\mathrm{dd}, J=5 \mathrm{~Hz}, J=11.2 \mathrm{~Hz}, H_{5}\right), 4.35\left(\mathrm{~s}, \mathrm{CH}_{3 \mathrm{Tr}}\right), 4.41\left(\mathrm{~d}, J=7.5 \mathrm{~Hz}, 1 \mathrm{H}, H_{1 \beta}\right), 4.87(\mathrm{sl}$, 
$3 \mathrm{H}, \mathrm{OH}), 5.05\left(\mathrm{dd}, J=15 \mathrm{~Hz}, J=20 \mathrm{~Hz}, 2 \mathrm{H}, H_{1}\right), 5.85\left(\mathrm{~s}, 2 \mathrm{H}, H_{4}\right), 7.50\left(\mathrm{~m}, 5 \mathrm{H}, H_{\text {arom }}\right), 8.72(\mathrm{~s}, 1 \mathrm{H}$, $H_{3}$ ) ${ }^{13} \mathrm{C}-\mathrm{NMR}\left(\mathrm{CD}_{3} \mathrm{OD}\right) 38.7\left(\mathrm{CH}_{3 \mathrm{Tr}}\right), 58.0,59.5\left(C_{1}, C_{4}\right), 66.9\left(C_{5}\right), 70.8,74.6,77.5\left(C_{2}, C_{3}, C_{4}\right)$, $104.6\left(C_{1 \beta}\right), 129.5\left(C_{3}\right), 129.8,130.0,130.5\left(C_{\text {arom }}\right), 133.4\left(C q_{\text {arom }}\right), 142.2\left(C_{2}{ }^{\prime}\right) .{ }^{19} \mathrm{~F}-\mathrm{NMR}\left(\mathrm{CD}_{3} \mathrm{OD}\right)$ $154.8\left(\mathrm{~s}, \mathrm{~B} F_{4}\right)$. Anal. Found (Calcd) for $\mathrm{C}_{15} \mathrm{H}_{19} \mathrm{~N}_{3} \mathrm{O}_{5}+1 \mathrm{H}_{2} \mathrm{O}: \mathrm{C} 43.96$ (43.56), $\mathrm{H} 5.18$ (5.48). $[\alpha]_{\mathrm{D}}{ }^{20}=-13.1(c 4.1 \mathrm{MeOH})$. HRMS calcd. for $\mathrm{C}_{16} \mathrm{H}_{22} \mathrm{~N}_{3} \mathrm{O}_{5}{ }^{+}: 336.1559$, found 336.1555

3.2.6. Preparation of 1-((1-Hexyl-3-methyl-1,2,3-triazol-4-yl)methoxy) $\beta$-D-xylopyranoside tetrafluoroborate (7)

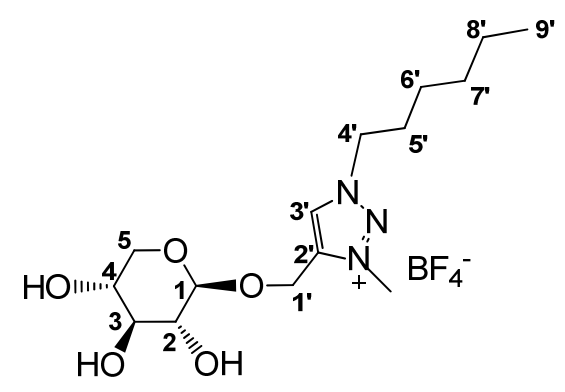

The same procedure as described for compound 6 was followed with the triazole 5 (900 $\mathrm{mg}$, $2.8 \mathrm{mmol}$ ) and $\mathrm{Me}_{3} \mathrm{OBF}_{4}(506 \mathrm{mg}, 3.4 \mathrm{mmol})$ in dry acetonitrile $(40 \mathrm{~mL})$. Compound 7 was obtained as as a white wax in $78 \%(\mathrm{~m}=896 \mathrm{mg})$. IR: $v \mathrm{~cm}^{-1}: 3392,3140,2956,2929,2872,2494,1589,1460$, 1356, 1323, 1286, 1247, 1038. ${ }^{1} \mathrm{H}-\mathrm{NMR}\left(250 \mathrm{MHz}, \mathrm{CD}_{3} \mathrm{OD}\right) 0.93$ (m, 3H, $H_{9}$ ), 1.37 (overlap, 6H, $\left.H_{6},+H_{7},+H_{8}\right), 2.01\left(\mathrm{~m}, 2 \mathrm{H}, H_{5}\right), 3.17-3.34$ (overlap, $\left.1 \mathrm{H}+1 \mathrm{H}+1 \mathrm{H}, H_{2}+H_{3}+H_{5}\right), 3.49(\mathrm{~m}, 1 \mathrm{H}$, $\left.H_{4}\right), 3.85\left(\mathrm{dd}, J=5 \mathrm{~Hz}, J=11.2 \mathrm{~Hz}, 1 \mathrm{H}, H_{5}\right), 4.32\left(\mathrm{~s}, 3 \mathrm{H}, \mathrm{CH}_{3 \mathrm{Tr}}\right), 4.37$ (d, $\left.J=7.5 \mathrm{~Hz}, 1 \mathrm{H}, H_{1 \beta}\right), 4.61$ (t, $\left.J=7.5 \mathrm{~Hz}, 1 \mathrm{H}, H_{4}{ }^{\prime}\right), 4.87\left(\mathrm{sl}, 3 \mathrm{H}, \mathrm{OH}\right.$ sugar), $5.03\left(\mathrm{dd}, J=7.5 \mathrm{~Hz}, J=22.5 \mathrm{~Hz}, 2 \mathrm{H}, H_{1}\right.$ ), 8.71 (s, $\left.1 \mathrm{H}, H_{3},\right) .{ }^{13} \mathrm{C}-\mathrm{NMR}\left(\mathrm{CD}_{3} \mathrm{OD}\right) 14.3\left(C_{9},\right), 23.4,26.7,30.1,32.1\left(C_{5}, C_{6}, C_{7}, C_{8^{\prime}}\right), 38.9\left(\mathrm{CH}_{3 \mathrm{Tr}}\right), 54.9$ $\left(C_{4},\right), 59.6\left(C_{1}\right), 67.0\left(C_{5}\right), 70.9,74.5,77.4\left(C_{2}, C_{3}, C_{4}\right), 104.8\left(C_{1 \beta}\right), 130.7\left(C_{3^{\prime}}\right), 142.0\left(C_{2}{ }^{\prime}\right) . \mathrm{NMR}^{19} \mathrm{~F}$ (235.4 MHz, $\left.\mathrm{CD}_{3} \mathrm{OD}\right) 155.1\left(\mathrm{~s}, \mathrm{~B} F_{4}\right)$. Anal. Found (Calcd) for $\mathrm{C}_{14} \mathrm{H}_{25} \mathrm{~N}_{3} \mathrm{O}_{5}+1.5 \mathrm{H}_{2} \mathrm{O}: \mathrm{C} 40.11$ (40.56), H 6.67 (7.03). [ $\alpha]^{20}{ }_{\mathrm{D}}=-20.4$ (c $4.4 \mathrm{MeOH}$ ). HRMS calcd. for $\mathrm{C}_{15} \mathrm{H}_{28} \mathrm{~N}_{3} \mathrm{O}_{5}{ }^{+}: 330.2029$, found 330.2033 .

\section{Conclusions}

D-Xylose-based ILs have been prepared from D-xylose following an original pathway, the key step being a click cycloaddition. These ILs have been fully characterized and are hydrophilic. After determination of their ecotoxicity and their biodegradability in a near future, these solvents could be used as alternative solvents or chiral agents for synthesis or catalysis in water under mild conditions.

\section{Acknowledgments}

This work was supported by the Fondation du Site Paris Reims (post doctoral fellowship for Nadège Ferlin) and the FEDER for material funds.

\section{Conflicts of Interest}

The authors declare no conflict of interest. 


\section{References}

1. Wasserscheid, P.; Welton, T. Ionic Liquids in Synthesis; Wiley-VCH-Verlag GmbH \& Co.: Weinheim, Germany, 2002.

2. Earle, M.J.; Esperança, J.M.S.S.; Gilea, M.A.; Lopes, J.N.C.; Rebelo, L.P.N.; Magee, J.W.; Seddon, K.R.; Widegren, J.A. The distillation and volatility of ionic liquids. Nature 2006, 439, 831-834.

3. Couling, D.J.; Bernot, R.J.; Docherty, K.M.; Dixon, J.K.; Maginn, E.J. Assessing the factors responsible for ionic liquid toxicity to aquatic organisms via quantitative structure-property relationship modeling. Green Chem. 2006, 8, 82-90.

4. Olivier-Bourbigou, H.; Magna, L.; Morvan, D. Ionic liquids and catalysis: Recent progress from knowledge to applications. Appl. Catal. A 2010, 373, 1-56.

5. Procuranti, B.; Myles, L.; Gathergood, N.; Connon, S.J. Pyridinium ion catalysis of carbonyl protection reactions. Synthesis 2009, 23, 4082-4086.

6. Myles, L.; Gore, R.; Spulak, M.; Gathergood, N.; Connon, S.J. Highly recyclable, imidazolium derived ionic liquids of low antimicrobial and antifungal toxicity: A new strategy for acid catalysis. Green Chem. 2010, 12, 1157-1162.

7. Van Rantwijk, F.; Sheldon, R.A. Biocatalysis in ionic liquids. Chem. Rev. 2007, 107, 2757-2785.

8. Plaquevent, J.-C.; Levillain, J.; Guillen, F.; Malhiac, C.; Gaumont, A.-C. Ionic liquids: New targets and media for $\alpha$-amino acid and peptide chemistry. Chem. Rev. 2008, 108, 5035-5060.

9. Welton, T. Room-temperature ionic liquids. Solvents for synthesis and catalysis. Chem. Rev. 1999, 99, 2071-2083.

10. Wei, D.; Ivaska, A. Applications of ionic liquids in electrochemical sensors. Anal. Chim. Acta 2008, 607, 126-135.

11. Buzzeo, M.C.; Evans, R.G.; Compton, R.G. Non-haloaluminate room-temperature ionic liquids in electrochemistry-A review. ChemPhysChem 2004, 5, 1106-1120.

12. Galinski, M.; Lewandowski, A.; Stepniak, I. Ionic liquids as electrolytes. Electrochim. Acta 2006, 51, 5567-5580.

13. Electrochemical Aspects of Ionic Liquids, 2nd ed.; Ohno, H., Ed.; John Wiley \& Sons, Inc.: Hoboken, NJ, USA, 2011.

14. Österholm, A.; Damlin, P.; Kvarnström, C.; Ivaskaa, A. Studying electronic transport in polyazulene-ionic liquid systems using infrared vibrational spectroscopy. Phys. Chem. Chem. Phys. 2011, 13, 11254-11263.

15. Liu, J.-F.; Jiang, G.-B.; Jönsson, J.A. Application of ionic liquids in analytical chemistry. TrAC Trends Anal. Chem. 2005, 24, 20-27.

16. Baker, G.A.; Baker, S.N.; Pandey, S.; Bright, F.V. An analytical view of ionic liquids. Analyst 2005, 130, 800-808.

17. Pandey, S. Analytical applications of room-temperature ionic liquids: A review of recent efforts. Anal. Chim. Acta 2006, 556, 38-45.

18. Soukup-Hein, R.J.; Warnke, M.M.; Armstrong, D.W. Ionic liquids in analytical chemistry. Annu. Rev. Anal. Chem. 2009, 2, 145-168. 
19. Sun, P.; Armstrong, D.W. Ionic liquids in analytical chemistry. Anal. Chim. Acta 2010, 661, 1-16.

20. Baltus, R.E.; Counce, R.M.; Culbertson, B.H.; Luo, H.; DePaoli, D.W.; Dai, S.; Duckworth, D.C. Examination of the potential of ionic liquids for gas separations. Sep. Sci. Technol. 2005, 40, 525-541.

21. Han, X.; Armstrong, D.W. Ionic liquids in separations. Acc. Chem. Res. 2007, 40, 1079-1086.

22. Arce, A.; Earle, M.J.; Katdare, S.P.; Rodriguez, H.; Seddon, K.R. Application of mutually immiscible ionic liquids to the separation of aromatic and aliphatic hydrocarbons by liquid extraction: A preliminary approach. Phys. Chem. Chem. Phys. 2008, 10, 2538-2542.

23. Luis, P.; Garea, A.; Irabien, A. Ionic liquids and membranes. J. Membr. Sci. 2009, 330, 80-89.

24. Gorri, D.; Ruiz, A.; Ortiz, A.; Ortiz, I. The use of ionic liquids as efficient extraction medium in the reactive separation of cycloolefines from cyclohexane. Chem. Eng. J. 2010, 154, 241-245.

25. Meindersma, G.W.; Hansmeier, A.R.; de Haan, A.B. Ionic liquids for aromatics extraction. Present status and future outlook. Ind. Eng. Chem. Res. 2010, 49, 7530-7540.

26. Leskinen, T.; King, A.W.T.; Kilpeläinen, I.; Argyropoulos, D.S. Fractionation of lignocellulosic materials with ionic liquids. Effect of mechanical treatment. Ind. Eng. Chem. Res. 2011, 50, 12349-12357.

27. Huisgen, R. 1,3-Dipolar cycloaddition. Proc. Chem. Soc. 1961, 357-396.

28. Himo, F.; Lovell, T.; Hilgraf, R.; Rostovtsev, V.V.; Noodleman, L.; Sharpless, K.B.; Fokin, V.V. Copper(I)-catalyzed synthesis of azoles. DFT study predicts unprecedented reactivity and intermediates. J. Am. Chem. Soc. 2005, 127, 210-216.

29. Rostovtsev, V.V.; Green, L.G.; Fokin, V.V.; Sharpless, K.B. A stepwise huisgen cycloaddition process: Copper(I)-catalyzed regioselective "ligation" of azides and terminal alkynes. Angew. Chem. Int. Ed. 2002, 41, 2596-2599.

30. Zekarias Yacob, Z.; Liebscher, J. 1,2,3-Triazolium Salts as a Versatile New Class of Ionic Liquids. "Ionic Liquids-Classes and Properties"; Handy, S.T., Ed.; InTech: Rijeka, Croatia, 2011; Chapter 1, pp. 1-22.

31. Jeong, Y.-K.; Kim, D.-Y.; Choi, Y.-S.; Ryu, J.-S. Intramolecular hydroalkoxylation in Brønsted acidic ionic liquids and its application to the synthesis of $( \pm)$-centrolobine. Org. Biomol. Chem. 2011, 9, 374-378.

32. Zhao, F.Q.; Xue, L.; Xing, X.L.; Hu, R.Z.; Zhou, Z.M.; Gao, H.X.; Yi, J.H.; Xu, S.Y.; Pei, Q. Thermochemical properties and thermokinetic behavior of energetic triazole ionic salts. Sci. China Chem. 2011, 54, 461-474.

33. Fletcher, J.T.; Keeney, M.E.; Walz, S.E. 1-Allyl- and 1-Benzyl-3-methyl-1,2,3-triazolium salts via tandem click transformations. Synthesis 2010, 26, 3339-3345.

34. Jeong, Y.; Ryu, J.-S. Synthesis of 1,3-Dialkyl-1,2,3-triazolium ionic liquids and their applications to the baylis-hillman reaction. J. Org. Chem. 2010, 75, 4183-4191.

35. Hollingsworth, R.I.; Wang, G. Toward a carbohydrate-based chemistry: Progress in the development of general-purpose chiral synthons from carbohydrates. Chem. Rev. 2000, 100, 4267-4282.

36. Murugesan, S.; Karst, N.; Islam, T.; Wiencek, J.M.; Linhardt, R.J. Dialkyl imidazolium benzoates-Room temperature ionic liquids useful in the peracetylation and perbenzoylation of simple and sulfated saccharides. Synlett 2003, 9, 1283-1286. 
37. Chiappe, C.; Marra, A.; Mele, A. Synthesis and applications of ionic liquids derived from natural sugars. Top. Curr. Chem. 2010, 295, 177-195.

38. Forsyth, S.A.; MacFarlane, D.R.; Thomson, R.J.; von Itzstein, M. Rapid, clean, and mild $O$-acetylation of alcohols and carbohydrates in an ionic liquid. Chem. Commun. 2002, 7, 714-715.

39. Rencurosi, A.; Lay, L.; Russo, G.; Caneva, E.; Poletti, L. Glycosylation with trichloroacetimidates in ionic liquids: Influence of the reaction medium on the stereochemical outcome. J. Org. Chem. 2005, 70, 7765-7768.

40. Murugesan, S.; Linhardt, R.J. Ionic liquids in carbohydrate chemistry-Current trends and future directions. Curr. Org. Synth. 2005, 2, 437-451.

41. Park, T.J.; Weiwer, M.; Yuan, X.J.; Baytas, S.N.; Munoz, E.M.; Murugesan, R.J. Linhardt. Glycosylation in room temperature ionic liquid using unprotected and unactivated donors. Carbohydr. Res. 2007, 342, 614-620.

42. Rosatella, A.A.; Frade, R.F.M.; Afonso, C.A.M. Dissolution and transformation of carbohydrates in ionic liquids. Curr. Org. Synth. 2011, 8, 840-860.

43. Galan, C.M.; Jones, R.A.; Tran, A.-T. Recent development of ionic liquids in oligosaccharide synthesis. The sweet side of ionic liquids. Carbohydr. Res. 2013, 375, 35-46.

44. Ogawa, C.; Kobayashi, S. Catalytic Asymmetric Synthesis, 3rd ed.; Wiley-VCH: Weinheim, Germany, 2010; p. 1.

45. Gaumont, A.-C.; Genisson, Y.; Guillen, F.; Plaquevent, J.-C. De Pasteur aux liquides ioniques chiraux. Petite histoire de l'induction asymétrique promue par le solvant. Actualité Chimique 2011, 348-349, 84-89.

46. Gaumont, A.-C.; Genisson, Y.; Guillen, F.; Zgonnik, V.; Plaquevent, J.-C. Chiral ionic liquids for asymmetric reactions. In Catalytic Methods in Asymmetric Synthesis; Gruttadauria, M., Giaccalone, F., Eds.: John Wiley \& Sons, Inc.: Palermo, Italy, 2011; pp. 323-344.

47. Chen, X.; Ying, A. Ionic liquids: Applications and perspectives. In Ionic Liquids: Applications and Perspectives; Kokorin, A., Ed.; InTech: Rijeka, Croatia, 2011; pp. 305-330.

48. Payagala, T.; Armstrong, D.W. Chiral ionic liquids: A compendium of syntheses and applications. Chirality 2012, 24, 17-53.

49. Ding, J.; Welton, T.; Armstrong, D.W. Chiral ionic liquids as stationary phases in gas chromatography. Anal. Chem. 2004, 76, 6819-6822.

50. Handy, S.T.; Okello, M.; Dickenson, G. Solvents from biorenewable sources: Ionic liquids based on fructose. Org. Lett. 2003, 5, 2513-2515.

51. Poletti, L.; Chiappe, C.; Lay, L.; Pieraccini, D.; Polito, L.; Russo, G. Glucose-derived ionic liquids: Exploring low-cost sources for novel chiral solvents. Green Chem. 2007, 9, 337-341.

52. Quadir, M.; Mathonneau, E. Cosmetic Composition Comprising Multiphasic Particles. U.S. Patent 20070231355, 4 October 2007.

53. Pereira, M.; Manuela, A. Chiral ionic liquids from carbohydrates: Synthesis and properties. Mini Rev. Org. Chem. 2012, 9, 243-260.

54. Truong, T.-K.-T.; van Buu, O.N.; Aupoix, A.; Pegot, B.; Vo-Thanh, G. Chiral ionic liquids derived from (-)-ephedrine and carbohydrates: Synthesis, properties and applications to asymmetric synthesis and catalysis. Curr. Org. Synth. 2012, 9, 53-64. 
55. Kumar, V.; Pei, C.; Olsen, C.E.; Schäffer, S.J.C.; Parmar, V.S.; Malhotra, S.V. Novel carbohydrate-based chiral ammonium ionic liquids derived from isomannide. Tetrahedron Asymmetry 2008, 19, 664-671.

56. Nguyen Van Buu, O.; Aupoix, A.; Vo-Thanh, G. Synthesis of novel chiral imidazolium-based ionic liquids derived from isosorbide and their applications in asymmetric aza Diels-Alder reaction. Tetrahedron 2009, 65, 2260-2265.

57. Nguyen Van Buu, O.; Aupoix, A.; Doan Thi Hong, N.; Vo-Thanh, G. Chiral ionic liquids derived from isosorbide: Synthesis, properties and applications in asymmetric synthesis. New J. Chem. 2009, 33, 2060-2072.

58. Ferlin, N.; Courty, M.; Gatard, S.; Spulak, M.; Quilty, B.; Beadham, I.; Ghavre, M.; Gathergood, N.; Bouquillon, S. Biomass derived ionic liquids: Synthesis from natural organic acids, characterization, toxicity, biodegradation and use as solvents for catalytic hydrogenation processes. Tetrahedron 2013, 69, 6150-6161.

59. Estrine, B.; Bouquillon, S.; Hénin, F.; Muzart, J. Telomerization of butadiene with L-arabinose and D-xylose in DMF: Selective formation of their monooctadienyl glycosides. Eur. J. Org. Chem. 2004, 2914-2922.

60. Estrine, B.; Bouquillon, S.; Hénin, F.; Muzart, J. Telomerization of butadiene with pentoses in water: Selective etherifications. Green Chem. 2005, 7, 219-223.

61. Hadad, C.; Damez, C.; Bouquillon, S.; Estrine, B.; Hénin, F.; Muzart, J.; Pezron, I.; Komunjer, L. Neutral pentosides surfactants issued from the butadiene telomerization with pentoses: Preparation and amphiphilic properties. Carbohydr. Res. 2006, 341, 1938-1944.

62. Damez, C.; Bouquillon, S.; Harakat, D.; Hénin, F.; Muzart, J.; Pezron, I.; Komunjer, L. Alkenyl and alkenoyl amphiphilic derivatives of D-xylose and their surfactant properties. Carbohydr. Res. 2007, 342, 154-162.

63. Deleu, M.; Damez, C.; Gatard, S.; Nott, K.; Paquot, M.; Bouquillon, S. Synthesis and physico-chemical characterization of bolaamphiphiles derived from alkenyl D-xylosides. New J. Chem. 2011, 35, 2258-2266.

64. Deleu, M.; Gatard, S.; Payen, E.; Lins, L.; Nott, K.; Flore, C.; Thomas, R.; Paquot, M.; Bouquillon, S. D-xylose-based bolaamphiphiles: Synthesis and influence of the spacer nature on their interfacial and membrane properties. C. R. Chim. 2012, 15, 68-74.

65. Hadad, C.; Majoral, J.P.; Muzart, J.; Caminade, A.M.; Bouquillon, S. First phosphorous D-xylose derived glycodendrimers. Tetrahedron Lett. 2009, 50, 1902-1905.

66. Camponovo, J.; Hadad, C.; Ruiz, J.; Cloutet, E.; Gatard, S.; Muzart, J.; Bouquillon, S.; Astruc, D. "Click" glycodendrimers containing 27, 81 and 243 modified xylopyranoside termini. J. Org. Chem. 2009, 74, 5071-5074.

67. Gatard, S.; Liang, L.; Salmon, L.; Ruiz, J.; Astruc, D.; Bouquillon, S. Water-soluble glycodendrimers: Synthesis and use in hydrogenation catalytic process. Tetrahedron Lett. 2011, $52,1842-1846$.

68. Hanelt, S.; Liebscher, J. A novel and versatile access to task-specific ionic liquids based on 1,2,3-Triazolium salts. Synlett 2008, 7, 1058-1060.

69. Shah, J.; Kahn, S.S.; Blumenthal, H.; Liebscher, J. 1,2,3-Triazolium-tagged prolines and their application in asymmetric aldol and Michael reactions. Synthesis 2009, 23, 3975-3982. 
70. Nakamura, T.; Ogata, K.; Fukuzawa, S.-I. Synthesis of dichlorobis(1,4-dimesityl-1H-1,2,3triazol-5-ylidene)palladium $\left[\mathrm{PdCl}_{2}(\mathrm{TMes})_{2}\right]$ and its application to suzuki-miyaura coupling reaction. Chem. Lett. 2010, 39, 920-922.

71. Nulwala, H.B.; Tang, C.N.; Kail, B.W.; Damodaran, K.; Kaur, P.; Wickramanayake, S.; Shi, W.; Luebke, D.R. Probing the structure-property relationship of regioisomeric ionic liquids with click chemistry. Green Chem. 2011, 13, 3345-3349.

72. Aizpurua, J.M.; Sagartzazu-Aizpurua, M.; Azcune, I.; Miranda, J.I.; Monasterio, Z.; García-Lecina, E.; Fratila, R.M. "Click” synthesis of nonsymmetrical 4,4'-Bis(1,2,3-triazolium) salts. Synthesis 2011, 17, 2737-2742.

73. Ohmatsu, K.O.; Kiyokawa, M.; Ooi, T. Chiral 1,2,3-Triazoliums as new cationic organic catalysts with anion-recognition ability: Application to asymmetric alkylation of oxindoles. J. Am. Chem. Soc. 2011, 133, 1307-1309.

74. Khan, S.S.; Shah, J.; Liebscher, J. Ionic-liquid tagged prolines as recyclable organocatalysts for enantioselective $\alpha$-aminoxylations of carbonyl compounds. Tetrahedron 2011, 67, 1812-1820.

75. Yoshida, Y.; Takizawa, S.; Sasai, H. Design and synthesis of spiro bis(1,2,3-triazolium) salts as chiral ionic liquids. Tetrahedron Asymmetry 2012, 23, 843-851.

76. Sanghi, S.; Willett, E.; Versek, C.; Tuominen, M.; Coughlin, E.B. Physicochemical properties of 1,2,3-triazolium ionic liquids. RSC $A d v$. 2012, 2, 848-853.

77. Mereyala, H.B.; Gurrala, S.R. A highly diastereoselective, practical synthesis of allyl, propargyl 2,3,4,6-tetra- $O$-acetyl- $\beta$-D-gluco- or $\beta$-D-galactopyranosides and allyl, propargyl heptaacetyl- $\beta$-Dlactosides. Carbohydr. Res. 1998, 307, 351-354.

78. Fischer, E. Ueber die verbindungen der zucker mit den alkoholen und ketonen. Ber. Dtsch. Chem. Ges. 1895, 28, 1145-1167.

79. Schulze, B.; Friebe, C.; Hager, M.D.; Günther, W.; Köhn, U.; Jahn, B.O.; Görls, H.; Schubert, U.S. Anion complexation by triazolium "ligands": Mono- and bis-tridentate complexes of sulfate. Org. Lett. 2010, 12, 2710-2713.

Sample Availability: Samples of the compounds 2-7 are available from the authors.

(C) 2013 by the authors; licensee MDPI, Basel, Switzerland. This article is an open access article distributed under the terms and conditions of the Creative Commons Attribution license (http://creativecommons.org/licenses/by/3.0/). 